\section{Familia y posmodernidad desde el existencialismo}

Family and postmodernity from an existential perspective
Centro Su

Social Science Journal Julio - Diciembre Vol 4 No 2 http://centrosureditorial.com/ind ex.php/revista elSSN: 2600-5743

revistacentrosur@gmail.com

Recepción: 1 marzo 2019 Aprobación 4 abril 2020

Pag 303 - 313

Atribución/ReconocimientoNoComercial-Compartirlgual 4.0 Licencia Pública Internacional CC BY-NC-SA 4.0 https://creativecommons.org/lice nses/by-nc-sa/4.0/legalcode.es

\section{Resumen}

El presente artículo parte del problema de investigación de la pérdida del fundamento en el campo intersubjetivo de la familia, en la época posmoderna dentro de países latinoamericanos; debido al aumento de la ruptura de relaciones matrimoniales, la crisis de la familia y la pareja, el abandono y el cuidado de los hijos por parte de terceros.

A partir de lo anterior, el objetivo de la investigación es comprender la pérdida del fundamento de la familia en la época posmoderna en países Latinoamericanos desde una perspectiva existencial.

Para esta investigación se trabajará desde una metodología de corte cualitativo, que permita explorar y comprender el fenómeno de la perdida de fundamento de la familia en la era de la posmodernidad.
Palabras
Clave:
familia, posmodernidad, existencialismo, crisis, Latinoamérica.

\author{
1. Magister. Corporación Universitaria Minuto de Dios; \\ Cafexistencial Centro de Estudios en Logoterapia e \\ Terapia Existencial. Soacha Colombia. Email. \\ Jose.mayorga@uniminuto.edu. ORCID: \\ https://orcid.org/0000-0002-0326-4824. Perfil de \\ Google Académico: \\ https://scholar.google.es/citations?user=7HeQZSMAAA \\ AJ\&hl=es
2 Magister. Corporación Universitaria Minuto de Dios. Email.mcaitaalvar@uniminuto.edu.co. ORCID: https://orcid.org/0000-0002-0345-4824. Perfil de Google Académico:
https://scholar.google.es/citations?user=7HeQZSMAAA $\mathrm{AJ} \& \mathrm{~K}=\mathrm{es}$




\section{Abstract}

The present article starts from the research problem of the loss of the foundation in the intersubjective field of the family, in the postmodern era within Latin American countries; due to the increase in the breakdown of marital relationships, the crisis of the family and the couple, the abandonment and care of children by third parties.

From the above, the objective of the research is to understand the loss of the family foundation in the postmodern era in Latin American countries from an existential perspective. For this research, we will work from a qualitative methodology that allows us to explore and understand the phenomenon of the loss of the family`s foundation in the postmodern.

Key words: Family, postmodernism, existentialism, crisis, Latin America.

\section{Introducción}

El presente artículo parte del problema de investigación de la pérdida del fundamento en el campo intersubjetivo de la familia, en la época posmoderna dentro de países latinoamericanos; debido al aumento de la ruptura de relaciones matrimoniales, la crisis de la familia y la pareja, el abandono y el cuidado de los hijos por parte de terceros.

A partir de lo anterior, el objetivo de la investigación es comprender la pérdida del fundamento de la familia en la época posmoderna en países Latinoamericanos desde una perspectiva existencial, dado que esta mirada logra comprender las relaciones humanas desde la posibilidad, como la confrontación con el otro, el mundo, el sí mismo y las situaciones como lo precisa Mayorga-González (2018).

Asimismo, parte de la pregunta de investigación es lograr responder ¿de qué manera se presenta la pérdida del fundamento de la familia en la época posmoderna en países Latinoamericanos desde una perspectiva existencial? Y para ello se vuelve de suma importancia indagar sobre este fenómeno en la era de la posmodernidad, luego, ser analizada desde la perspectiva teórica anteriormente dicha.

Para esta investigación se trabajará desde una metodología de corte cualitativo, que permita explorar y comprender el fenómeno de la perdida de fundamento de la familia en la era de la posmodernidad, utilizando como instrumento de recolección de datos la rejilla bibliográfica, con el fin de identificar las posturas actuales ante este fenómeno y ser comprendidas desde el análisis documental. 
Con todo lo anterior, a manera de hipótesis se podría llegar a considerar que la familia ha perdido el fundamento posiblemente a causa de los objetivos de la posmodernidad que llegan a generar una ruptura y disolución con otros campos, restringiendo de esta forma, su interés en la protección y la reproducción de la especie. Por el contrario, si esto no se presenta de esa manera, el fundamento de la familia no se encontraría en relación con la época posmoderna desde la mirada existencial.

A partir de lo anterior, a continuación, se profundizará sobre la familia, la era de la posmodernidad, la perspectiva existencial y luego su relación teórica, para poder realizar a través del análisis documental la categorización y reflexión en torno a nuestro objetivo de investigación.

\section{Familia}

Es necesario delimitar el concepto de familia, entiendo esta como un campo intersubjetivo que da lugar a la formación de la realidad y se limita por normas, roles y funciones (Sotolongo, 2006). A partir de estos roles, se orienta el funcionamiento y establecimiento de la familia como campo en el que se origina la manera en la que el individuo se desenvuelve en los diversos contextos. Asimismo, y en apoyo al planeamiento anterior, (Laing, 1969) en su libro El cuestionamiento sobre la familia plantea la familia como un sistema, es decir, como relaciones entre sus miembros y no como elementos aislados unos de otros.

Por otro lado, cuando se hace referencia al campo de la familia es de suma importancia hacer énfasis en el matrimonio y cómo éste ha pasado por fuertes cambios y transformaciones a lo largo del tiempo. Según afirma Coontz (2005), el matrimonio se encuentra en una crisis generalizada que, si bien se atribuye a múltiples causas, todas derivan en vínculos matrimoniales y relacionales frágiles. Así, los individuos se enfrentan a relaciones inconsistentes e inestables que dan lugar a fragmentaciones emocionales que posteriormente repercuten en los diversos campos en los que se desenvuelve en su vida cotidiana.

En este sentido, los roles que desempeñan los miembros de la familia se han modificado paulatinamente, por lo que el fundamento de la familia ha sido atenuado por nuevas prácticas y distintos esquemas en la estructura, conformación y forma de relacionarse dentro de la familia. Así, es importante realizar una revisión exhaustiva que permite un acercamiento a la comprensión del impacto de la era posmoderna en la familia como campo intersubjetivo que se plantea desde una mirada existencial.

Posmodernidad 
La época actual se encuentra enmarcada por distintas transiciones y múltiples cambios percibidos en la forma de comportarse y relacionarse de los individuos, que apartan o dejan de lado valores y normas aceptadas socialmente por mucho tiempo, así como también dan lugar a nuevos planteamientos de la manera en la que el ser humano debería asumir su lugar en el mundo (De Castro, 2000).

Así pues, la posmodernidad está caracterizada por aspectos puntuales que determinan y orientan los roles que fundamentan el campo de la familia, dentro de los cuales se encuentra el consumo, el comercio, la política y la tecnología, entre otros (Mayorga-Gonzalez, 2020); de modo que modifican las relaciones entre hombres y mujeres, entre padres e hijos, entre hermanos y entre las variadas formas de relacionarse en los múltiples campos.

En consecuencia, la disolución de la relación entre el sujeto y el campo de la familia, se atribuye a la disminución de compromiso en lo que respecta a la reproducción y a la responsabilidad de vivir junto a otra persona, dejando de lado intereses, gustos y placeres personales (Mayorga-Gonzalez, 2020). Así, se retoma la idea planteada anteriormente en la que se afirma que la crisis del matrimonio sumada a la perdida de cargas morales, culturales, sociales y legales; repercuten directamente en la base de la construcción de la familia.

En este orden de ideas, Coontz (2005) asegura que las esferas de actividades desempeñadas tanto por hombres como por mujeres se han disuelto, por lo que sus roles tanto en el mundo como en la familia, se encuentran en una evidente transición; lo que lleva al cambio en el rol madre, el rol padre y a su vez una profunda variación en el fundamento de la familia.

\section{Perspectiva existencial}

El contenido existencial de la cotidianidad de los individuos se origina en la relación con el otro, puesto que, de esta forma, tal y como lo plantea Heidegger (1980), el sujeto solo existe en la presencia del otro; y es su relación con el otro la que da sentido y significado a su existencia. Así, el existir como padre o madre, solo es posible y solo tiene sentido cuando se tiene un hijo; el docente existe conjuntamente con el estudiante, y de esta forma con las demás relaciones que se presentan en los diferentes campos.

En este sentido, la familia es un "nosotros común" que para Laing (1969) está en contraposición a un "ellos"; por tanto es de esperar una relación recíproca en el momento en el que cada sujeto se identifica como una parte de aquél nosotros. Dicho ejercicio es sumamente importante puesto que da lugar a la comprensión de un pensamiento colectivo, así como el sentido de hacer parte a aquel nosotros. 
Apoyando lo anterior, se hace énfasis en el contexto, el rol y los otros, como elementos de la dinámica relacional que dan lugar a la construcción de la realidad. Así mismo, se menciona la situación como elemento importante ya que posibilita la relación entre el mundo interno y externo (Mayorga-Gonzalez, 2018). Por ende, los elementos puestos en consideración se reúnen en la búsqueda de la auto-confirmación y la confirmación por los otros.

Como bien se ha mencionado, la familia como campo intersubjetivo, estructurado a partir de las relaciones entre los miembros de la misma, los ritus (patrones recurrentes), lo sacro (fundamentos) y el logos (posibilidades); se nutre del pensamiento colectivo y de la construcción de un nosotros, tal como lo señala Mayorga-Gonzalez (2020), aspectos que se han perdido en la posmodernidad puesto que se ha hecho común la individualidad, la búsqueda de la satisfacción personal, la evasión de compromisos a largo plazo y eludir la responsabilidad que la reproducción trae consigo.

Por ello, y como se sugiere en momentos anteriores, los roles de los miembros de la familia presentan gran tendencia a ser cambiantes, dinámicos e inestable. Algunos de ellos buscan salir de dicho campo, dejando vacios que de una u otra forma deben suplirse y ser asumidos por algún otro miembro de la familia, nombrados por MayorgaGonzalez (2020) como figuras simbólicas.

De esta forma se hace clara la manera en la que se pierde el equilibrio del campo y cómo para los miembros de la familia no es posible en muchas ocasiones llegar a encontrar su lugar en el mundo, su lugar en la familia y hacerse cargo de su propia existencia. Esto posteriormente trae grandes repercusiones en los numerosos campos en los cuales se encontrará a lo largo de su vida.

A partir de lo anterior, Mayorga-Gonzalez (2020) reconoce la importancia de asumir la propia formación y el reconocimiento de la propia existencia como adultos emocionalmente responsables, capaces de orientar y servir de guías para los otros, así como de ocupar el lugar que les corresponde. Esto implica, reconocer y ser concientes de las posibilidades de cambio que se evidencian en las situaciones a las que se enfrenta el individuo.

\section{Materiales y métodos}

Se hace uso del método cualitativo, entendido este como el enfoque metodológico que comprende la recolección de datos sin medición numérica con el fin de llegar a la interpretación profunda de significados (Hernández, Fernández y Baptista, 2010). Así pues, los datos cualitativos tienden a ser descripciones sumamente detalladas, bien sea de situaciones, eventos, personas, conductas o interacciones. 
En este sentido, Hernández, Fernández y Baptista (2010) mencionan el patron cultural como un punto importante a tener en cuenta, siendo un común denominador del la interpretación cualitativa en el que toda cultura o sitema social tiene un método particular para comprender las situaciones.

Asimismo, por medio de la rejilla bibliográfica, como instrumento de recolección de datos, se busca identificar las posturas actuales ante el fenómeno y ser comprendidas desde el análisis documental; que se considera como un proceso mediante el cual se facilita el acceso, consulta y recuperación de la información de manera concisa y resumida, bien sea de acuerdo con su contenido o a su forma (García, 1993).

De igual manera, en torno a la conceptualización de este instrumento de recolección de información se destacan dos posturas: en la primera de ellas, el análisis documental corresponde a diversas fases que incluyen la descripción bibliográfica y la catalogación; y, por otro lado, la segunda mirada hace énfasis en que el análisis documental solo debe pensarse como el análisis de contenido del tema en cuestión (García, 1993).

Autores como Dulziades y Molina se refieren al análisis documental como "una forma de investigación técnica, un conjunto de operaciones intelectuales, que buscan describir y representar los documentos de forma unificada sistemática para facilitar su recuperación" (2004). Además de esto, señalan que el análisis documental es útil en cuanto permite la organización y el fácil acceso de diversas fuentes para su posterior uso; así como constituye una vía que beneficia el acercamiento de los sujetos a la información.

En este sentido, la importancia y utilidad del análisis documental radica en su valor como herramienta o instrumento de trabajo a partir de la facil recuperación y acceso a diversas fuentes bibliográficas en torno a un o unos temas puntuales, de manera ordenada y estructurada. En síntesis, el análisis y la investigación documental, tal y como la plantea Tancara (1993), se refiere a "una serie de métodos y técnicas de búsqueda, procesamiento y almacenamiento de la información contenida en los documentos en primera instancia" (p. 94).

Así, para el presente artículo, la rejilla da lugar a trece documentos, dentro de los cuales se dividen de la siguiente manera: 7 artículos, 4 libros y 2 tesis y/o trabajos de grado; obtenidos de diferentes bases de datos. Para facilitar su comprensión, se recurre al uso de categorías y subcategorías (como se evidencia en los resultados), esto con el fin de facilitar la condensación de la información encontrada. 


\section{Resultados}

De acuerdo a los documentos evidenciados en la rejilla se plantean cuatro categorías que se irán desarrollando a lo largo de este apartado, con el fin de comprender los temas en cuestión; cabe resaltar que dichas cateogrías se encuentras estrechamente relacionadas unas con otras, por lo que resulta complicado entenderlas de manera aislada.

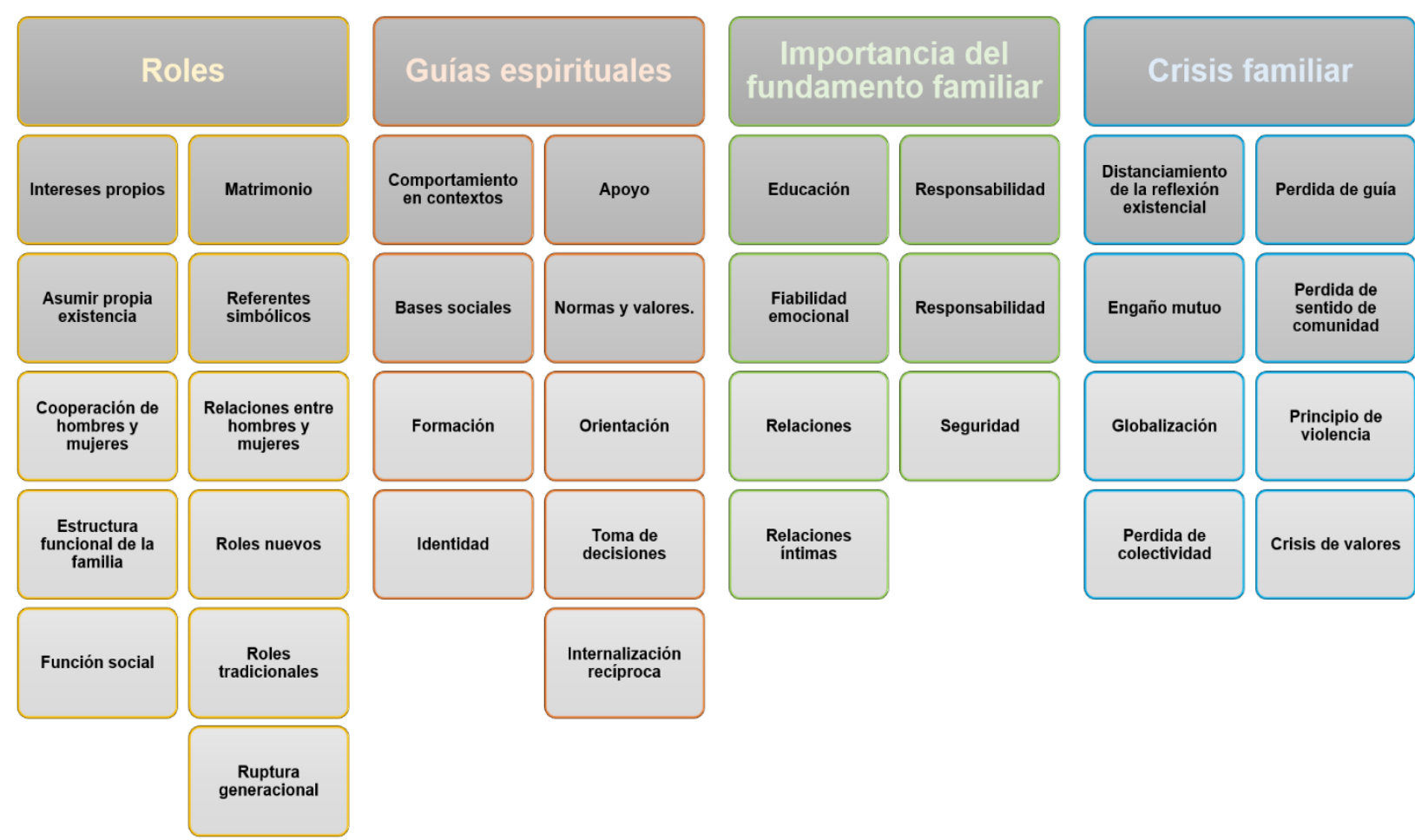

En primer lugar se evidencia que los diferentes roles que asume cada individuo en los diversos campos y en específico en el campo intersubjetivo de familia, ha sufrido múltiples cambios y variaciones, dejando de lado los roles tradiconales y dando lugar a nuevos roles (Mayorga-Gonzalez, 2018), así, es claro cuando Rivera (2014) plantea una ruptura generacional que se traduce en la ausencia de ayuda y cooperación entre hombres y mujeres.

Dando continuidad a lo anterior, queda claro que las relaciones entre hombres y mujeres ocupan un lugar fundamental en la estrutura funcional de la familia. Tal como lo plantea Coontz (2005) y Mayorga-González (2020), esto da lugar a la transformación del papel que cumple el matrimonio dentro del campo familiar; así, lo que en épocas anteriores sería predecible, hoy por hoy se encuentra en proceso disyuntivo y cambiante; entonces se encuentra que las funciones que tenía el matrimonio y la familia, está siendo reemplazado por otras instituciones. 
Dicho cambio, fundado en nuevas prácticas y nuevos esquemas económicos, políticos y reproductivos que demandan el cambio de las relaciones tradicionales, hombre y mujeres se encuentran orientados a romper con los rígidos patrones de género en los que se les tenía encasillados y a optar por intereses propios. Lo anterior, claramente ha tenido gran incidencia en la funcion de hombres y mujeres en el campo familiar, social, laboral, económico, político, personal, entre otros.

Si se hace una aproximación a la familia en cuanto a su estructura funcional y teniendo en cuenta el planeteamiento previo, la relación entre la familia, como campo primario de socialización del niño (Cooper, 1994) y la educación hacia niños y jóvenes, pone en tela de juicio los valores sociales, que claramente requieren una fuerte reconstrucción (Salón, 2010); así como también hace que se cuestione y reflexione acerca de los referentes simbólicos, cuyos valores se han idealizado a fin de obtener seguridad a nivel individual y social (Díaz, 2009).

Al pensar en cómo ha cambiado y evolucionado la familia, no se puede dejar de lado la reflexión personal y la responsabilidad que tiene cada individuo de asumir su propia existencia de manera real para permitirse fluir, siendo capaces de sentir y de amar positivamente para sí mismos y para otros (Mayorga-Gonzalez, 2020).

Una persona que se hace cargo de su propia existencia y que conoce su lugar en el mundo y su lugar en la familia, será un persona idónea para ocupar el papel de referente simbólico, lo que a su vez, lo dota de cualidades y capacidades en lo que respecta a servir de guía espiritual para otros, sabiendo todo lo que esto conlleva.

Stolorow, Atwood y Oragne, hacen énfasis en como la familia enseña de diferentes manera y en diferentes momentos cuáles son aquellos comportamiento entendidos como bueno o malos, así como aquellos que son convenientes en los diferentes contextos (2002).

Álvarez en el 2014, hace un aporte fundamental a cerca de como el posmodernismo no posee una "etiqueta identitaria", como pasaba en épocas anteriores donde se generaba en torno al género o al grupo familiar. Esto se puede traducir como falta de internalización recíproca por parte de sus miembros, tal como lo diría Sartre (citado por Laing, 1969), que se refiere a la ausencia de la concepción de familia como un nosotros y no como un ellos.

Por otro lado, recordando la familia como la base social, se hace visible como las familias multiproblemáticas aumentan, como los padres evaden sus responsabilidad de educación con sus hijos, como dicha responsabilida pasa a ser de terceros y como muchos de estos infantes crecen sin tener guías claros ni herramientas para tomar decisiones (Fort y Plaza, 2018). En concordancia con lo anterior, Rodríguez en su 
artículo titulado, La familia posmoderna: Distancia y compromiso, pone en manifiesto la existencia de una "gran discapacidad entre normas y valores donde fuimos socializados y las exigencias actuales" (2001, p. 113).

\section{Discusión}

Así pues, es importante realizar una reflexión en lo que respecta a la importancia del fundamento familiar, que corresponde a la tercera categoría propuesta; en este punto se reconocer diferentes puntos importantes que se irán desarrollando transitoriamente. Por un lado, las relaciones se fundan en gran medida, en torno a la fiabilidad emocional que se pueda encontrar en el otro (Coontz, 2005) que a su vez determina las capacidades que cada individuo desarrolla para servir al otro dentro del campo familiar:

Lo anterior, da lugar a la búsqueda constante de seguridad tanto a nivel individual como a nivel social (López, 2001). Así, Laing (1969) afirma que las relaciones familiares requieren que cada uno de los miembros asuma reciprocamente en su interior la imagen de unos nosotros. De no ser así, puede que las relaciones íntimas se ven claramente afectadas y dichas personas no logren alcanzar una educación para alcanzar un contacto real consigo mismas y con el otro (González-Mayorga, 2020). Finalmente, a partir del recorrido documental realizado, surgue entonces la cuarta categoría, correspondiente a los factores que influyen, determinan y se derivan de la crisis del fundamento de la familia en tiempos de posmodernidad. El distanciamiento de los indivuos con ejercicios de reflexión existencial implica el enfrentamiento ante inseguridades para responder a las actividades de los diversos campos y evitan que los individuos asuman su ugar enb el mundo de forma responsable (Jiménez, 2011).

Con esto, diversos procesos tales como la globalización encuentran en dichas falencias personales y familiares una forma de ejercer control y manipular con fines económicos y políticos (Álvarez, 2014), nos encontramos ante una cultura individualista y a una fractura en el pensamiento colectivo y cooperador, regidos, como lo plantea Mayoga-González (2018), bajo el engaño mutuo, el principio de violencia y la crisis generalizada de valores orientadores para encontrarse a sí mismo, para encontrar el lugar en la familia y el lugar en el mundo.

\section{Conclusiones}

La familia dada como campo intersubjetivo en el que se dan los primeros procesos de socialización y acercamiento a los otros, juega un papel fundamental, cuya importancia determina y modifica todos los campos en los que los individuos se van a encontrar a lo largo de su vida. Por esto, es vital recobrar los valores y dar el lugar que dicho campo requiere, pero, que se ha perdido con el paso del tiempo y 
especialmente en la época posmoderna. Como se trato a lo largo de dicho documento, tal perdida se atribuye a diversos factores como el cambio en los roles tanto de hombres como mujeres, así como la ruptura de los individuos con procesos vitales referidos a la reflexión existencial. En este sentido, el afán de la cotidianidad y las exigencias de sociedades capitalistas y consumistas, llevan a los individuos a un pensamiento individualista y superficial, olvidando la importancia del sentir colectivo, crítico y profundo. Esto último, da cabida a la pérdida del sentido de responsabilidad por parte de los sujetos, consigo mismos y con los otros; dicho de otro modo, aquella persona que en ningún momento asumió la responsabilidad de su propia existencia, será un guía equívoco para otros y no contará con las herramientas adecuadas para servir como referente simbólico. Entonces, nos encontramos en un ciclo que se reproduce en múltiples generaciones; de allí la importancia y la invitación a generar procesos de reflexión responsables.

\section{Referencia}

Álvarez Cabrales, D., y Nieves Oviedo, J. A. (2014). Crisis de las identidades posmodernas y la búsqueda de la colectividad en el espacio (Doctoral dissertation, Universidad de Cartagena).

Coontz, S. (2005). Historia del matrimonio. Barcelona: Editorial Gedisa.

Cooper, D. (1994). La muerte de la familia. Planeta-De Agostini,.

De Castro, A. (2000). La Psicología existencial de Rollo May. Ediciones Uninorte. Barranquilla.

Díaz, Y. L. (2009). Familia, querida familia, ¿hacia dónde vas?. Trabajo Social, (11).

Dulzaides, I. ME; Molina G. AM. 2004. Análisis documental y de información: dos componentes de un mismo proceso. Revista SciELO, 12(2), 1-2.

Fort, J. T., \& Plaza, Y. M. (2018). Sociedad, familia y escuela en la Postmodernidad. Interacciones turbulentas, relativismo y anomia. Análisis. Revista Colombiana de Humanidades, 50(93), 427-447.

García, A. (1993). Análisis documental: el análisis formal. Revista general de información y documentación, 3(1), 11.

Heidegger, M (1980). Hölderlins Hymnen "Germanien" und "Der Rhein". Suzanne Ziegler (ed.). Frankfurt am Main: Vittorio Klostermann (Gesamtausgabe vol. 39)

Hernández-Sampieri, R., Fernández, C., y Batista, P. (2010). Metodología de la Investigación. 5ta. Edición. México: Editorial Mc Graw Hill. Hernández, C. 
Jiménez, M (2011). Capitulo 4. Ronald David Laing. En Martínez, Y, \& Signorelli, S. (2011). Perspectivas en Psicoterapia Existencial. Ediciones LAG. México.

Laing, R. D. (1969). El cuestionamiento de la familia. Paidós.

Mayorga-Gonzalez, J. (2020). La construcción de la relación de pareja en la mujer posmoderna: Una aproximación compleja y existencial en la vida cotidiana. México.

Mayorga-Gonzalez, J. (2020). Mi lugar en la familia. Cafexistencial.

Mayorga-Gonzalez, J. M. (2018). Dinámica relacional en familias emergentes. III Bienal Latinoamericano y Caribeña de Infancias y Juventudes: Desigualdades, desafios a las democracias, memorias y re-existencias, 419-424.

Rivera, R. (2014). El valor simbólico de las mujeres, visto desde la perspectiva de la masculinidad mexicana contemporánea. Pacarina del Sur. Revista de Pensamiento Crítico Latinoamericano

Rodríguez, A. (2001). La familia posmoderna: distancia y compromiso. Revista Redes, (8).

Salón, R. R. (2010). Juventud, familia y posmodernidad:(des) estructuración familiar en la sociedad contemporánea. Fermentum. Revista Venezolana de Sociología y Antropología, 20(57), 39-55.

Sotolongo, P. (2006). Teoría social y vida cotidiana, La Habana, Acuario.

Stolorow R, Atwood G y Orange D, (2002), Mundos de Experiencia: Un Concepto Fundamental de la Teoría Psicoanalítica de la Intersubjetividad, Pág. (19-38)

Tancara, C. (1993). La investigación documental. Temas sociales, (17), 91-106. 